\title{
Minimizing Counterexample of ACTL Property
}

\author{
ShengYu Shen, Ying Qin, and SiKun Li \\ School of Computer Science, \\ National University of Defense Technology of China \\ \{syshen, qy123, skli\}@nudt.edu.cn
}

\begin{abstract}
Counterexample minimization tries to remove irrelevant variables from counterexamples, such that they are easier to be understood. For the first time, we proposes a novel approach to minimize looplike and path-like counterexamples of ACTL properties. For a counterexample $s_{0} \ldots s_{k}$, our algorithm tries to extract a succinct cube sequence $c_{0} \ldots c_{k}$, such that paths run through $c_{0} \ldots c_{k}$ are all valid counterexamples. Experimental result shows that our algorithm can significantly minimize ACTL counterexamples. 1
\end{abstract}

\section{Preliminaries}

BDD contain two terminal nodes and a set of variable nodes. Attribute value(u) is associated with terminal nodes $u$. Every variable node has two outgoing edges: llow $(\mathbf{u})$ and $\operatorname{high}(\mathbf{u})$. A variable $\operatorname{var}(\mathbf{u})$ is associated with every node $u$.

Symbolic model checking with BDD is first proposed by K.McMillan [1, which is implemented by procedure Check that takes a CTL formula and returns BDD of those states that satisfy the formula.

Assume the state variable set of Kripke structure $M=\langle S, I, T, L\rangle$ is $V=$ $\left\{v_{0}, \ldots, v_{n}\right\}$. A state $s \in S$ can be seen as assignments to $V$, which is denoted by $s=\left\{v_{0} \leftarrow b_{0}, \ldots, v_{n} \leftarrow b_{n}\right\}$, with $b_{i} \in\{0,1\}$ are boolean constant. Assume $V^{\prime}=\left\{v_{i_{0}}, \ldots, v_{i_{m}}\right\}$ is a subset of $V$, then projection of s to $V^{\prime}$ is defined as

$$
\left.s\right|_{V^{\prime}}=\left\{v_{i_{0}} \leftarrow b_{i_{0}}, \ldots, v_{i_{m}} \leftarrow b_{i_{m}}\right\}
$$

A state set $S^{\prime} \subseteq S$ is a cube iff there exists $V^{\prime}=\left\{v_{i_{0}}, \ldots, v_{i_{m}}\right\} \subseteq V$ and $\left\{b_{i_{0}}, \ldots, b_{i_{m}}\right\}$, such that $S^{\prime}==\left\{s|s|_{V^{\prime}}==\left\{v_{i_{0}} \leftarrow b_{i_{0}}, \ldots, v_{i_{m}} \leftarrow b_{i_{m}}\right\}\right\}$

Assume state $s$ is in state set $S^{\prime}$, then $c$ is a cube guided by $s$ in $S^{\prime}$ iff $s \in c \subseteq S^{\prime}$. We denote $c$ by Guided $\operatorname{Cube}\left(\mathbf{S}^{\prime}, \mathbf{s}\right)$, it can be computed as below.

\section{Algorithm 1: Computing GuidedCube $\left(S^{\prime}, s\right)$}

1. Assume $s=\left\{v_{0} \leftarrow b_{0}, \ldots, v_{n} \leftarrow b_{n}\right\}$.

2. $c=\phi \quad V^{\prime}=\phi$ are all empty set

3. $c n=$ root node of BDD of $S^{\prime}$

${ }^{1}$ Supported by Chinese NSF under Grant No.90207019 and No.60403048; the Chinese 863 Program under Grant No. 2002AA1Z1480. 
4. while( $c n$ isn't a terminal node)
(a) assume $\operatorname{var}(\mathrm{cn})$ is $v_{i}$
(b) if $\left(b_{i}==0\right)$ then $c n=\operatorname{low}(\mathrm{cn})$ else $c n=\operatorname{high}(\mathrm{cn})$
(c) $c=c \cup\left\{v_{i} \leftarrow b_{i}\right\} \quad V^{\prime}=V^{\prime} \cup\left\{v_{i}\right\}$

5. GuidedCube $\left(S^{\prime}, s\right)=\left\{s^{\prime}\left|s^{\prime}\right|_{V^{\prime}}==c\right\}$

\section{Minimizing Counterexample of ACTL Property}

Existing approaches [2] can only deal with path-like counterexamples of invariant $A G f$. For the first time, this paper proposes a novel approach to minimize looplike and path-like counterexamples of ACTL properties. Due to duality of ACTL and ECTL, we will focus on minimizing witness of ECTL formula.

To make a witness $s_{0} \ldots s_{k}$ more easy to be understood, some state variables must be removed. So a minimized witness must be a cube sequence $c_{0} \ldots c_{k}$. We define the criteria that it must satisfied.

Definition 1 (Criteria of Minimized Witness of ECTL Property). Assume $s_{0} \ldots s_{k}$ is a witness of an ECTL property $f$. Cube sequence $c_{0} \ldots c_{k}$ is the minimized witness of $s_{0} \ldots s_{k}$ iff

1. $s_{i} \in c_{i}(0 \leq i \leq k)$

2. Every path $s_{0}^{\prime} \ldots s_{k}^{\prime}$ that satisfy $\bigwedge_{0 \leq i \leq k} s_{i}^{\prime} \in c_{i}$ must be witness of $f$

We will discuss minimizing witness of $E X, E U$ and $E G$ below.

\subsection{Minimizing Witness of EX and EU}

Assume PreImage $\left(S^{\prime}\right)$ is a procedure that computes pre-image of $S^{\prime}$. We can minimize $E X f$ witness $s_{0} s_{1}$ and $E[f U g]$ witness $s_{0} \ldots s_{k-1}$ in the following way:

\section{Algorithm 2: Minimizing Witness of $E X f$}

1. $c_{1}=$ GuidedCube $\left(\right.$ Check $\left.(f), s_{1}\right)$

2. $c_{0}=$ GuidedCube $\left(\right.$ PreImage $\left.\left(c_{1}\right), s_{0}\right)$

\section{Algorithm 3: Minimizing Witness of $E[f U g]$}

1. $c_{k-1}=$ GuidedCube $\left(\right.$ Check $\left.(g), s_{k-1}\right)$

2. for $i=k-2$ to 0

3. $\quad c_{i}=$ GuidedCube $\left(\right.$ PreImage $\left(c_{i+1}\right) \cap$ Check $\left.(f), s_{i}\right)$

Correctness proof is omited due to space limitation.

\subsection{Minimizing Witness of EG}

A loop-like witness of $E G f$ contains two segments: a stem $s_{0} \ldots s_{m}$ and a loop $s_{m} \ldots s_{n}$. We will first prove the following theorem below. 
Theorem 1. Assume a loop-like witness of EGf contains two segments: a stem $s_{0} \ldots s_{m}$ and a loop $s_{m} \ldots s_{n}$. Then a cube sequence $c_{0} \ldots c_{n}$ is its minimized witness if the following 4 equations hold true

$$
\begin{gathered}
\bigwedge_{0 \leq i \leq n} s_{i} \in c_{i} \\
\bigwedge_{n} \subseteq \operatorname{PreImage}\left(c_{m}\right) \wedge \bigwedge_{\substack{0 \leq i \leq m-1 \\
\bigwedge_{i \leq m-1}}} c_{i} \subseteq \operatorname{PreImage}\left(c_{i+1}\right) \\
\bigwedge_{0 \leq i \leq n} c_{i} \subseteq \operatorname{Preck}(f)
\end{gathered}
$$

Proof. By equation (2), the 1st criteria of Definition 1 is satisfied.

Assume a path $s_{0}^{\prime} \ldots s_{n}^{\prime}$ satisfy $T\left(s_{n}^{\prime}, s_{m}^{\prime}\right) \wedge \bigwedge_{0 \leq i \leq n} s_{i}^{\prime} \in c_{i}$. By equation (5), $\bigwedge_{0 \leq i \leq n} M, s_{i}^{\prime}=f$.

Thus this theorem is proven.

We compute an approximation of $c_{m} \ldots c_{n}$ with following algorithm.

\section{Algorithm 4: $\operatorname{Min}(x)$}

1. $c_{m}=x$

2. $c_{n}=$ GuidedCube $\left(\right.$ PreImage $\left(c_{m}\right) \cap$ Check $\left.(f), s_{n}\right)$

3. For $i=n-1$ to $m$

4. $\quad c_{i}=$ GuidedCube $\left(\right.$ PreImage $\left(c_{i+1}\right) \cap$ Check $\left.(f), s_{i}\right)$

5. return $c_{m}$

To compute $c_{m} \ldots c_{n}$ that satisfies equation (3), we first let

$$
C=C h e c k(E G f)
$$

And then run $\operatorname{Min}(C)$. Cube sequence $c_{m} \ldots c_{n}$ obtained in this way satisfies almost all $\subseteq$ relation in equation (3) , except $c_{n} \subseteq \operatorname{PreImage}\left(c_{m}\right)$.

So we need to run Algorithm 4 iteratively, and obtain the following sequence:

$$
\operatorname{Min}(C), \operatorname{Min}^{2}(C), \ldots \operatorname{Min}^{t}(C), \ldots
$$

We terminate above iteration only when $\operatorname{Min}^{t-1}(C) \subseteq \operatorname{Min}^{t}(C)$, at which $c_{n} \subseteq$ PreImage $\left(c_{m}\right)$ and equation (3) can be satisfied. So we must prove that iteration in equation (7) is terminable with following theorems.

Theorem 2. Min $(x)$ is monotonic. (Proof is omited due to space limitation)

Theorem 3. $C \supseteq \operatorname{Min}(C)$ 
Table 1. Experimental Result

\begin{tabular}{|c|c|c|c|c|c|}
\hline Cex name & Cex length & \multicolumn{2}{|c|}{ Original cex } & \multicolumn{2}{c|}{ Minimized cex } \\
\cline { 3 - 6 } & & $\begin{array}{c}\text { Number of. } \\
\text { Variables. }\end{array}$ & $\begin{array}{c}\text { Run } \\
\text { time }\end{array}$ & $\begin{array}{c}\text { Number of } \\
\text { Variables }\end{array}$ & $\begin{array}{c}\text { Run } \\
\text { time }\end{array}$ \\
\hline P1 & 13 & 1027 & 0.12 & 244 & 0.12 \\
\hline P2 & 7 & 308 & 0.01 & 172 & 0.02 \\
\hline L1 & 64 & 975 & 0.991 & 791 & 1.45 \\
\hline L2 & 76 & 1140 & 1.26 & 942 & 1.96 \\
\hline L3 & 75 & 1125 & 2.83 & 929 & 4.09 \\
\hline L4 & 22 & 858 & 0.19 & 510 & 0.24 \\
\hline L5 & 22 & 858 & 0.28 & 467 & 0.33 \\
\hline L6 & 22 & 858 & 0.16 & 455 & 0.17 \\
\hline L7 & 22 & 858 & 0.12 & 408 & 0.17 \\
\hline
\end{tabular}

Proof. By Algorithm 4, for every state $s_{m}^{\prime} \in \operatorname{Min}(C)$, there is a path $s_{m}^{\prime} s_{m+1}^{\prime} \ldots s_{n}^{\prime} s_{m} "$, such that $s_{m} " \in C$. That is to say, there is an infinite path $p$ starting from $s_{m}$ ", and $f$ holds true at all states along $p$.

By Algorithm 4, $f$ holds true on all states of $s_{m}^{\prime} s_{m+1}^{\prime} \ldots s_{n}^{\prime} s_{m}$ ".

Thus, we can concatenate $s_{m}^{\prime} s_{m+1}^{\prime} \ldots s_{n}^{\prime} s_{m}$ " and $p$, to form a new path $p^{\prime} . f$ hold true at all states along $p^{\prime}$. Thus, $p^{\prime}$ is witness of $M, s_{m}^{\prime} \models E G f$.

By equation (6), we can conclude that $s_{m}^{\prime} \in C$.

Thus, $C \supseteq \operatorname{Min}(C)$ is proven.

Theorem 4. The iteration in equation (7) is terminable.

Proof. By Theorem 2 and 3 , it is obvious that : $C \supseteq \operatorname{Min}(C) \ldots \supseteq \operatorname{Min}^{t}(C) \ldots$

So $\exists$ t. $\operatorname{Min}^{t-1}(C)==\operatorname{Min}^{t}(C)$ hold true. Thus, this theorem is proven.

Thus, we can construct minimized witness $c_{m} \ldots c_{n}$ in the following way:

\section{Algorithm 5: Minimizing Witness of $E G f$}

1. $c_{m}=\operatorname{Min}^{t}(C)$

2. $c_{n}=$ GuidedCube $\left(\right.$ PreImage $\left(c_{m}\right) \cap$ Check $\left.(f), s_{n}\right)$

3. for $i=n-1$ to 0

4. $\quad c_{i}=$ GuidedCube $\left(\right.$ PreImage $\left(c_{i+1}\right) \cap$ Check $\left.(f), s_{i}\right)$

\section{Experimental Result}

We implement our algorithm in NuSMV, and perform experiments on NuSMV's benchmarks. All experiments run on a PC with $1 \mathrm{GHz}$ Pentium 3.

Table 1 presents experimental result. The 1 st column lists the name of counterexamples. P1 and P2 are path-like counterexamples. All others are loop-like counterexamples. The 2nd column lists their length. The 3rd column lists the number of variables in original counterexamples. The 4 th column lists the time taken by NuSMV to generate these counterexamples. The 5 th column lists the 
number of variables in minimized counterexamples. The last column lists the run time of our approach.

From the experimental result, it is obvious that our algorithm can significantly minimize counterexamples.

\section{References}

1. K.L.McMillan. Symbolic model checking. Kluwer Academic Publishers, 1993.

2. K. Ravi and F. Somenzi. Minimal assignments for bounded model checking. In TACAS'04,LNCS 2988, pages 31-45, 2004. 